\title{
Conflictos socioambientales o los escenarios urgentes para las ciencias sociales ecuatorianas en el Siglo XXI
}

Pablo Ortiz-T*

Hace poco se presentó en un acto público una trilogía de libros referentes a "Prevención y Tratamiento de Conflictos Socioambientales y Políticas Públicas". La publicación es el resultado de un esfuerzo compartido, colectivo y puntual entre la Universidad Politécnica Salesiana, a través de docentesinvestigadores de la carrera de Gestión para el Desarrollo Local, el equipo de técnicos de la Subsecretaría de Diálogo Social de la Secretaría de Pueblos, Movimientos Sociales y Participación Ciudadana -la principal entidad auspiciantey Editorial Universitaria Abya-Yala. A esa tarea se incorporaron investigadores de otras entidades como el Instituto de Altos Estudios Nacionales (IAEN), la Facultad Latinoamericana de Ciencias Sociales (FLACSO), la Universidad Andina Simón Bolívar (UASB) y las fundaciones ECOCIENCIA y Futuro Latinoamericano (FFLA).

Más allá de una novedad de estantería esta publicación invita a reflexionar y debatir en torno a los procesos conflictivo-socioambientales en marcha en el país, a los requerimientos conceptuales, metodológicos e institucionales alrededor de una gestión o tratamiento de los conflictos asociados a la explotación o aprovechamiento de bienes de la naturaleza, que estén acordes a la vigencia y garantía plena de derechos humanos fundamentales (tanto individuales como colectivos) y en particular al rol del Estado y el tipo de respuestas que éste brinda. Precisamente ese campo de exigencias nos remite a un ejercicio de exploración de posibles hipótesis o respuestas tentativas a varias interrogantes centrales presentes en este debate: ¿Cómo dar cuenta de manera objetiva a la dinámica de conflictos socioambientales? ¿Cuál es el locus desde el cuál es posible aquello? ¿Son adecuadas las herramientas conceptuales y metodológicas que se utilizan para tratar conflictos en condiciones de diversidad cultural, exclusión y gran asimetría? ¿En qué medida la forma como se tratan estos conflictos fortalece la capacidad de control del Estado sobre los territorios y las poblaciones locales?

Candidato doctoral. Máster en Ciencias Políticas. Docente e investigador de la UPS, Carrera de Gestión para el Desarrollo Local 
La búsqueda de respuestas a éstas y otras interrogantes demanda ahora y en el futuro, mayores análisis y reflexiones de la comunidad académica, pero también de los diferentes protagonistas e involucrados en las conflictividades. De manera rápida en el presente texto se plantean algunos elementos de reflexión conceptual, de alguna manera complementarios a los ya planteados en los textos, con referencia central a los conflictos socioambientales y al panorama complejo que plantea Ecuador en este campo, lo cual sin dudase constituye en un reto no solo para la investigación y el quehacer académico, sino y fundamentalmente, para la propia sociedad y el Estado en el marco de su búsqueda de reformas y cambios al orden social existente.

\section{La pertinencia de la Teoría Social}

Abordar los conflictos sociales, como objeto específico de atención e investigación es un ejercicio relativamente reciente en la agenda académica, pese a su antigua y estrecha vinculación con las relaciones sociales, la dinámica de los cambios históricos, y el haber formado parte de objetos ineludibles de análisis en la historiografía social, en las Ciencias Políticas, en las teorías de relaciones internacionales y en las mismas teorías sociológicas.

En las teorías sociales, por ejemplo, los conflictos sociales estaban relacionados al problema del orden y de la integración social, versus el problema del cambio y la desarticulación social (Bhargava, 1992). En el primer caso, basta aludir a Rousseau y su planteamiento de la sociedad como un todo armónico en el que la integración social debía ser el resultado de consensos. Y en el segundo caso, la idea de la discordia social, frente a lo cual es posible la integración sobre la base de la coacción social, en una perspectiva claramente hobbesiana, en la que consideró que el poder del hombre reside en su capacidad de actuar y la adquisición del poder se convierte en una búsqueda permanente y dominada por la pasión. La persona actúa según los impulsos que recibe del exterior, por lo que intentará a toda costa evitar los impulsos que le resulten desagradables y conseguir todos los agradables posibles. El problema surge cuando estas fuentes de placer hay que compartirlas con otros humanos o interfieren con sus deseos (Bobbio, 1985). Esto determina que cada ser humano esté en continua guerra con los demás. Esta situación en la que vive el hombre en su estado na- 
tural encontró su mejor definición en dos de sus sentencias más universalmente conocidas: Bellum omnium contra omnes ("Guerra de todos contra todos") y Homo homini lupus ("El hombre es un lobo para el hombre").

En este proceso de análisis del humano y sus sentidos, llega a una serie de definiciones que serán cruciales para la filosofía de Hobbes. Bobbio recuerda la importancia de estas definiciones, insinuando que está intentando axiomatizar la humanidad siguiendo el modelo de la geometría. Esta influencia de las ciencias exactas se percibe en la manera tan objetiva y carente de sentimiento en la que describe las pasiones; por ejemplo, "Lo que de algún modo es objeto de cualquier apetito o deseo humano es lo que con respecto a él se llama bueno. Y el objeto de su odio y aversión, malo; y de su desprecio, vil e inconsiderable o indigno. Pero estas palabras de bueno, malo y despreciable siempre se usan en relación con la persona que las utiliza. No son siempre y absolutamente tales, ni ninguna regla de bien y de mal puede tomarse de la naturaleza de los objetos mismos, sino del individuo (donde no existe Estado) o (en un Estado) de la persona que lo representa; o de un árbitro o juez a quien los hombres permiten establecer e imponer como sentencia su regla del bien y del mal" (Hobbes, 2005:42). ${ }^{1}$

Hobbes encontró tres motivos básicos por los cuales hay conflictos en el Estado de Naturaleza: el primero, es la competición, que hace que el hombre invada para obtener algo; el segundo, la desconfianza para la seguridad; y el tercero, la gloria, para la reputación. De estos tres conceptos partirán las leyes de naturaleza que Hobbes las clasifica en 19; sin embargo, la primera y segunda ley son las más importantes y de ellas se van a deducir todas las demás. La primera ley se compone de dos partes: Cada hombre debe procurar la paz hasta donde tenga esperanza de lograrla; y cuando no puede conseguirla, entonces puede buscar y usar todas las ventajas y ayudas de la guerra. La segunda parte ser refiere al derecho natural a la libertad de cada hombre, que lo autoriza de usar su propio poder, según le plazca, para la preservación de su propia vida, y, por lo tanto, de hacer cualquier cosa que conciba como la más adecuada para

1 Puede ampliarse sobre este punto en el capítulo VI del Origen Interno de las ociones Voluntarias, Comúnmente llamadas pasiones y términos por medio de los cuales se expresan. Hobbes (2005: 40$50)$. 
alcanzar ese fin. De esta ley se va a derivar la segunda ley: Un hombre debe estar deseoso, cuando otros lo están también, y a fin de conseguir la paz y la defensa personal hasta donde le parezca necesario, de no hacer uso de su derecho a todo, y de contentarse con tanta libertad en su relación con los otros hombres, como la que él permitiría a los otros en su trato con él. De aquí en adelante, las leyes de Hobbes van a definir el contrato social (Bobbio, 1985)²

Hobbes desarrolla su idea del contrato o pacto social, desarrollado por los hombres como garantía de la seguridad individual y como forma de poner fin a los conflictos que, por naturaleza, generan estos intereses individuales. Así, a las pasiones naturales del hombre se oponen las leyes morales, siendo a su vez leyes naturales. El Estado (o República) que Hobbes proyecta en Leviatán no es el concepto moderno de república (ausencia de monarquías) sino que es concebido como una res publica, es decir, un poder organizado de forma común cuya función es 'regentar' las cosas públicas y que se funda a partir de la suma de voluntades individuales libres que deciden actuar para adquirir ventajas comunes. La libertad del individuo se verá reducida a los espacios donde la ley no se pronuncia. Estas premisas serán pilares centrales en el pensamiento político occidental.

Precisamente los liberales consideraron las actividades estatales dentro de una sociedad de modos más o menos sofisticados, como lo hizo Adam Smith (1987) en su tercer libro La Riqueza de las Naciones en el cual describió el modo en que la extensión del comercio, al permitir a la aristocracia feudal gastar su dinero en mercancías y no en servidores, permitió que se impusiera el imperio de la ley. La cadena causal del argumento de Smith era compleja y sutil: la fragmentación de la soberanía que tuvo lugar después de la caída de Roma, es decir, un factor político, fue lo que dio lugar a la ciudad autónoma y productiva que tanto influyó económicamente para socavar el poder feudal (Grathoff, 1978; Bhargava,

2 Hay que anotar que Bobbio al analizar el pensamiento hobbesiano, llama la atención sobre aquella premisa según la cual el individuo asocial del estado de naturaleza que vive en continua sospecha de ser engañado y ofendido por los demás, que no respeta las leyes de la naturaleza por temor a que otros las transgredan antes que él, perpetuamente asediado por la voluntad de hacer daño. Es la base de ese Iusnaturalismo, que expresa la naturaleza egoísta del ser humano, cuya premisa central considera que éste no es apto para asociarse por naturaleza, sino llega a serlo mediante la educación" cfr. Hobbes, 1985, 153 y ss. 
1992). Esa versión de Smith entendía cabalmente que el poder tiene sus propios atractivos, que es susceptible del abuso y que está en permanente necesidad de ser controlado, y por eso su insistencia en que un cierto tipo de Estado, un "vigilante nocturno" minimalista, proporcionaba la mejor cobertura para el crecimiento económico. Creía que se "necesita poco más que paz, impuestos bajos y una tolerable administración de justicia para llevar al Estado desde la barbarie más abyecta al más elevado grado de opulencia; todo lo demás es producido por el curso natural de las cosas" (Smith, 1980: 322).

Esas premisas estuvieron presentes con fuerza en todo el pensamiento de los liberales, quienes tenían presente que los Estados habían obtenido gran parte de su preeminencia gracias a sus actividades externas. El pensador más sofisticado en esa dirección sin duda fue Immanuel Kant (2002) y su 'paz perpetua' que tiene una característica realista singular, en tanto aceptaba que el Estado, dada la 'sociedad asocial' de las relaciones dentro de la Europa de su época, era un instrumento de seguridad necesario. Si los Estados tenían gobiernos liberales, se abrían a los extranjeros y potenciaban el comercio con otros Estados similares en una liga liberal, y de ahí surgiría la paz. Los Estados serían refrenados por quienes pudieran morir en una guerra, y la conciencia generalizada de que las ventajas mutuas de las interrelaciones comerciales serían destruidas por la guerra resultaría igualmente importante. Una de las dificultades del argumento de Kant es que es problemático especificar con precisión qué factor previene la guerra; otra es la sospecha de que un Estado liberal podría ver un rival no liberal como una afrenta total a su modo de vida, algo que en realidad podría intensificar un conflicto geopolítico (Holst, 2002).

Estas ya antiguas preocupaciones serían abordadas dos siglos más tarde por otros autores como Max Weber, Karl Marx hasta Charles Wright Mills, Tom Bottomore, Alain Touraine, Ralph Dahrendorf o Anthony Giddens, quienes en general se inscribirían en una suerte de línea conflictivista de análisis, y que permiten entender distintas tipologías múltiples, según se trate de variables y unidades de estudio como estados, estructuras económicas, ideologías, instituciones, grupos raciales, étnicos, etáreos, entre otros. Precisamente dos de los autores mencionados, Dahrendorf y Giddens abordarían el tema de la conflictividad remitiéndose a autores clásicos como Marx y sus teoría de las clases sociales. 
En el caso de Ralph Dahrendorf en su ya clásico texto Clases y Conflictos de Clase en la Sociedad Industrial ${ }^{3}$, evalúa las contribuciones marxistas a una teoría del conflicto social y de clases. Toma distancia del mismo, al menos en sus versiones más esquemáticas y dogmáticas, y resalta que un aporte fundamental de la teoría materialista de la historia fue reconocer que los conflictos sociales son permanentes e inherentes al funcionamiento de la sociedad, aunque advertía que oponer únicamente dos grupos únicos con intereses totalmente opuestos (proletariado y burguesía) reduce o representa una simplificación extrema, aunque en principio reconoce que la teoría marxista de las clases es interesante como fondo histórico, en tanto serán las clases (sociales) las fuerzas actuantes en los conflictos sociales. Dahrendorf reconoce que la propiedad de los medios de producción y la pertenencia a una clase social, pueden ir disociados en las sociedades del capitalismo contemporáneo. Según este autor alemán, de hecho, el control de los medios económicos de producción constituye un caso particular de las relaciones generales de dominio. Esquemáticamente plantea que un empresario y un asalariado miembros del mismo club deportivo, en el que poseen el mismo grado de autoridad, pertenecerían a una misma clase social situacionalmente. En última instancia, lo que determina el conflicto no es la propiedad legal, sino el control real de los medios de producción y -particularmente- en manos de burócratas sin propiedad alguna. De esta manera, Dahrendorf va a desplazar el análisis de la realidad económica de la propiedad a la realidad vinculada al sistema de poder (1979:127 y ss.).

En esa dirección Dahrendorf (1992) sugiere que el quid estructural del conflicto social es la desigual distribución de la autoridad entre personas y grupos de la sociedad. Piensa que la distribución de la autoridad es, además de radicalmente desigual, dicotómica, existiendo incluso el estado de privación absoluta de ella. El conflicto social se explicaría, entonces, a partir de la dualidad extrema de sus oponentes; las estructuras de autoridad constituyen la causa determinante de la constitución de clase y de los conflictos de clase.

3 La versión original se publicó en la Universidad de Stanford en 1959, "Class and Class Conflict in Industrial Society”, Stanford University Press. La versión española (utilizada en nuestro presente artículo) está publicada en 1979. 
Para poder indagar acerca del conflicto social y orientar una relación significativa con la existencia de clases sociales, Dahrendorf acentuará la presencia de grupos de interés (stakeholders) en la sociedad. Los caracteriza a éstos como agrupaciones que poseen una organización, un programa de acción e intereses definidos, es decir, con iguales directrices conscientes de conducta (intereses manifiestos). Por otro lado, se presentarán los cuasi-grupos. Claramente distintos de los primeros, serán subgrupos que comparten intereses latentes derivados de una situación común de sus constituyentes elementales, sin que estos tengan necesariamente conciencia de ello (vecinos, colectividades, clubes sociales, centros culturales, estudiantes, etcétera. Todos los conflictos serán, pues, conflictos de autoridad: choques entre grupos de intereses, uno de los cuales defiende cada uno de los elementos de lo existente o su combinación -status quo-, mientras que el otro exige su modificación. Sindicatos, partidos políticos, movimientos sociales, serán ejemplos de los grupos de interés, quienes desencadenarán el conflicto al concretar las razones de las contradicciones y radicalizar la acción de los subgrupos.

La importancia metodológica del concepto de clase social que propone Dahrendorf basado en la autoridad, en cuanto instrumento sociológico, radica en la aplicación práctica de la teoría de clases en ámbitos empíricos concretos, permitiéndonos reconocer una pluralidad indeterminada de clases, de modo que podamos identificar dominantes y dominados en cualquier asociación (situación estructural) que posea una mínima distribución de la autoridad (Dahrendorf, 1990: 193 y ss.).

En el caso de Anthony Giddens (1994), luego de evaluar las exposiciones teóricas de autores fundacionales como Weber y Marx, realizará un replanteo conceptual en torno al mercado capitalista y a la capacidad de mercado de los individuos, que posteriormente empleará a lo largo de su trabajo, y siguiendo esos mismos conceptos desembocará en su conclusión y punto de partida para la confección de una nueva teoría de la estructuración de las relaciones de clases: “...el problema (...) no estriba en reconocer la diversidad de las relaciones y conflictos creados por el mercado capitalista como tal, sino en llevar a cabo la transición teórica de dichas relaciones y conflictos a la identificación de las clases como formas estructurales" (Giddens, 2006: 115). Para Giddens es imperativo abandonar la identificación del concepto de clase con las divisiones e 
intereses que genera el mercado. En ese marco, aun cuando pueda existir una multiplicidad indefinida de intereses intersectoriales engendrados por las diferentes capacidades del mercado, solo existe, en una sociedad dada, un número limitado de clases (Giddens, 1994: 119). Se torna prioritario en ese esquema desenganchar el concepto de clase de su dimensión o determinación exclusivamente económica. En principio, para este autor una clase es un agregado en gran escala de individuos compuesto por relaciones definidas impersonalmente y nominalmente abierto en su formas (1994: 120). Para complementar esta definición, retomará la perspectiva weberiana de clase social, entendidas como un conjunto de situaciones de clase vinculadas entre sí por el hecho de que encierran posibilidades comunes de movilidad bien dentro de la profesión de los individuos o a través de las generaciones. A partir de allí, estructuración de clases, conflicto social, y movilidad social, serán una tríada inseparable en la teoría de Giddens, quien distinguirá dos tipos de estructuración de clases: la estructuración mediata y la estructuración inmediata (2006: 122 y ss.)

En el primer caso alude a factores que intervienen entre la existencia de unas cualidades de mercado dadas y la formación de clases como grupos sociales identificables, operando las últimas como formas de relación 'total' entre el mercado y los sistemas estructurados de relación de clase. Adicionalmente este tipo de estructuración coloca en relación directa las capacidades de mercado con las posibilidades de movilidad social. Mientras más cerradas sean las clases, mayores las posibilidades de estructuración. Por eso Giddens afirma que, la estructuración de clases se ve facilitada en la medida en que el cierre de la movilidad existe en relación a cualquier forma específica de capacidad de mercado (Giddens,2006: 121).

En cambio en la estructuración inmediata, existen tres fuentes de estructuración relacionadas: la división del trabajo, el sistema de autoridad y la influencia de grupos de consumo. La división del trabajo facilita la formación clases al crear grupos homogéneos. Al mismo tiempo, la influencia de la técnica industrial crea una separación decisiva entre trabajadores manuales y no-manuales. Aquello se traslapa con la influencia de la estructuración mediata a través de la distribución desigual de las posibilidades de movilidad y es, a su vez, reforzado en la medida en que el sistema de autoridad típico de la empresa, a través de las normas de autoridad, separa a los trabajadores de cuello blanco de los traba- 
jadores manuales. En el ámbito jerárquico, los factores de control y propiedad -que había introducido Dahrendorf- son útiles para visualizar una diferenciación en las cúpulas entre clase alta y media.

Finalmente analiza la influencia de los denominados grupos distributivos, entendidos como aquellos que se producen a partir de relaciones que entrañan formas comunes de consumo de determinados bienes o servicios. Estos grupos, son importantes en la medida que se relacionan con los factores antes citados, de forma tal que refuercen las separaciones típicas entre las formas de capacidad de mercado. Para Giddens los grupos distributivos más significativos son, “(...) aquellos formados a través de la tendencia a la segregación por comunidades o barrios" (Giddens, 2006: 124). En pocas palabras, el primer proceso de estructuración especifica la forma en que se estructuran las definiciones y localizaciones de clase. Mientras que el segundo, define e impone un límite a dichas localizaciones. La combinación de ambos tipos de estructuración da lugar al sistema de estratificación triple en la sociedad capitalista.

Sin lugar a dudas la teoría social aporta elementos clave para el análisis y tratamiento de los conflictos de distinto tipo, en tanto uno de los núcleos centrales a comprender refiere a la constitución y naturaleza de las partes en disputa, sean clases implicadas, estamentos, grupos de interés (stakeholders) cuyas dinámicas de cambio estructural y posicionamiento coyuntural deben ser considerados al momento de analizar y discutir tipos de salidas posibles a los conflictos presentados.

\section{EI reto de comprender la conflictividad socioambiental}

Estos complejos marcos teóricos -al menos en sus partes centrales- sin duda están presentes en los usos de muchos autores como Johan Galtung (1996) o Peter Wallesteen (2007) que abocan conocimiento de campos conflictivos específicos, de manera especializada. Otro de esos ejemplos que podría citarse es el caso del investigador canadiense Thomas Homer-Dixon, quien incursiona con fuerza en la teorización y análisis de los llamados conflictos socioambientales. ${ }^{4}$

$4 \quad$ Al respecto cfr. Pablo Ortiz-T. Aproximación conceptual a los conflictos socioambientales (2001: 17 y ss). 
El hecho de que estos conflictos sociales se puedan calificar o no de socioambientales no es relevante, ni siquiera analíticamente. Lo importante es saber si estos conflictos califican como tales (cumpliendo condiciones para existir como esos fenómenos), si conducirán a otro tipo de eventos conflictivos, si lo harán o derivarán en situaciones de mayor violencia y se afrontará o no un problema de seguridad en el sentido clásico del término, en función de su impacto sobre la sociedad y no tanto sobre la naturaleza.

Entre los especialistas como Thomas Homer-Dixon (1991), Peter Gleick (1993), Thomas Naff y Ruth Matson (1984) o Peter Wallesteen (2007) no existe ninguna clase de acuerdo en cuanto a vincular directamente problemas ambientales y conflicto. Existen no muchas evidencias de que ciertos problemas ambientales -más bien de carácter global- como los que menciona Homer-Dixon (1999), tales como efecto invernadero, agujero en la capa de ozono, depósito de ácidos, deforestación, degradación de la tierra cultivable, polución y escasez de las reservas de agua o reducción/agotamiento de los recursos pesqueros puedan generar una serie de efectos en cadena como pueden ser:

a. Reducción de la capacidad productiva del campo.

b. Empeoramiento de la situación económica de los países afectados.

c. Desplazamiento de poblaciones.

d. Trastornos en el funcionamiento de comunidades e instituciones (crisis de gobernabilidad del Estado).

El que los problemas medioambientales deriven en conflicto o no depende de dos condiciones centrales: por un lado, de las capacidades de los Estados para adaptarse a los problemas sociales y económicos que éstos generan; y por otro lado, de las comunidades y poblaciones afectadas (directa o indirectamente). Adicionalmente, autores como Michael Renner (1996) advierten de los cambios en las nociones de seguridad, que en términos contemporáneos se abre hacia nuevas dimensiones, lo cual coloca sobre la mesa la necesidad de analizar riesgos que antes se habían mantenido en un segundo plano de interés, y la forma en que estos riesgos podían afectar o provocar conflictos entre actores nacionales e internacionales. 
Si bien es verdad que ni el riesgo ambiental ni el conocimiento de su existencia son recientes, también es cierto que la percepción del riesgo ha cambiado desde los años setenta. Así, se ha tomado conciencia del carácter limitado de los recursos de la biosfera y de la desigualdad en su distribución y acceso; de la dimensión global de los riesgos, que no respetan las fronteras; de las distintas prioridades y perspectivas de los países industrializados y los del llamado 'Tercer Mundo'; y de la insuficiencia de las soluciones técnicas y, por ello, de la necesidad de adoptar soluciones políticas ante dichos riesgos.

Las posibles respuestas a los riesgos medioambientales acostumbran a estar ligadas al desarrollo socioeconómico, por lo que los gobiernos como el de Ecuador en la actualidad se enfrentan a decisiones difíciles: frenar o invertir las tendencias de desarrollo (basado en un esquema extractivo) o promover inversiones en opciones de desarrollo alternativas, o ralentizar el potencial para la cooperación multilateral en la protección medioambiental hasta que se hayan alcanzado los objetivos de desarrollo propios (tal como se plantea con la llamada Iniciativa Yasuní-ITT) (Ortiz, 2011).

Las recientes negociaciones globales en torno a las amenazas asociadas al calentamiento global y al cambio climático, evidencian que la dinámica dominante a nivel global es esta tercera, con lo que se sientan las bases para futuros conflictos (Giddens, 2010, Terán, 2011).

Por otra parte, también se ha apreciado que los desafíos medioambientales que pueden provocar mayor conflictividad son aquéllos relacionados con la escasez de recursos como agua, tierra apta para la agricultura, los bosques nativos, los recursos pesqueros (Gleick, 1993, Renner, 1996). La conciencia de la existencia de riesgos medioambientales ligados a la carestía de recursos condujo en las últimas tres décadas al análisis, y el debate sobre el mismo, hacia la relación de los riesgos medioambientales con conflictos sociales, y particularmente aquellos que adquieren connotaciones violentas. Una ventaja para el debate es que el análisis de esta relación entre medio ambiente y conflictos está aún muy poco desarrollado, con lo que el material y los autores a estudiar son relativamente accesibles y precisamente ahí radica el aporte para el contexto ecuatoriano de los textos de la Universidad Politécnica Salesiana, la Secretaría de Pueblos y Editorial Universitaria Abya-Yala que sin duda motivan, introdu- 
cen y aproximan, especialmente a los interesados y neófitos en este apasionante y complejo campo de investigación-acción.

\section{Desafíos a futuro en el Ecuador}

Si el año 2010 -según una publicación del Programa de Derechos Humanos de la Universidad Andina Simón Bolívar- en el Ecuador se registraron 268 conflictos relacionados con derechos humanos referidos al Buen Vivir, libertades y derechos de los pueblos y nacionalidades (PADH, 2011), habría que preguntarse si en realidad se trataron de conflictos, de denuncias de atropellos, de problemas estructurales preexistentes o de problemáticas nuevas, más allá de manifestar una problemática y una preocupación permanente con la que muchos coincidimos: si los casos de conflictividad degeneran en violaciones a las libertades, el derecho a la vida, la integridad personal, la libertad de expresión, los derechos de los pueblos y en particular, lo que aluden como tensión entre la defensa que pueblos indígenas realizan de su territorio y la naturaleza y la política extractivista del Estado", considerando que estos sujetos colectivos son además sujetos de derechos amparados en la Constitución.

Una revisión exhaustiva de casos permite constatar que en Ecuador, un factor desencadenante de conflictos que aparece cada vez más con mayor frecuencia es el interés de distintos actores por controlar y aprovechar determinados tipos de bienes de la naturaleza. El mayor porcentaje de los conflictos citados en el estudio del PADH (2011) tienen que ver con el rechazo y oposición de comunidades locales a actividades extractivas en minería, dada la amenaza que estas actividades representan para los recursos hídricos (Ortiz, 2011). Parecería que los conflictos, según esta publicación, están asociados a la denuncias de grupos activistas (algunas alrededor de un mismo caso) más que a procesos conflictivos en sí mismos.

La pregunta a formularse es si esa dinámica de conflictos es novedosa en el Ecuador, se diría que no, en tanto serán los años 90 del siglo anterior los que marcan el desate de conflictos socioambientales -recuérdense los conflictos en torno a los impactos derivados de las actividades petroleras en la Amazonía, o a la expansión de la frontera camaronera y la depredación de los bosques de manglar en la Costa- y que tuvieron desde entonces como variable deter- 
minante, sea en el objeto en disputa o en las motivaciones o intereses de los grupos de interés, el control, aprovechamiento o gestión de la naturaleza y sus recursos. Asociar mecánicamente modelo de desarrollo primario-exportador o modelo extractivo y dinámica de conflictos, es arriesgado, porque dicho modelo está articulado a la formación histórico-social del país, y no necesariamente su constitución y consolidación ha significado una constante en la existencia de ese tipo de conflictos, como tampoco lo ha sido el comportamiento de los distintos actores involucrados, tanto del lado del Estado, de las empresas privadas como de las organizaciones sociales, campesinas e indígenas. Los conflictos simplemente varían de una época a otra, y de un lugar a otro. Incluso un mismo actor o grupo de actores involucrados no necesariamente tiene el mismo tipo de reacción frente a otro tipo de conflicto.

De ahí que cobran importancia estas publicaciones para encontrar hilos conductores para presentes y futuras investigaciones que posibiliten profundizar los temas, analizarlos, debatirlos. Solo la investigación y el debate profundo permitiría tener un panorama objetivo y claro, donde no sean los prejuicios ni las extrapolaciones superficiales las que supriman la necesidad de investigar y analizar. Precisamente en el trabajo aludido que se acaba de presentar por parte de la UPS, en el segundo volumen de la trilogía, denominado Conflictos socioambientales, políticas públicas y derechos. Aproximación a un debate, es escrito por Víctor Hugo Torres, María José Narváez, Víctor López Acevedo, Volker Frank, Pippa Heyling, Susan Engel, Jackie Rivera, Miguel Castro, Karla Beltrán y Juan Calles López, quienes abordan un mosaico de temas que plantean experiencias prácticas y específicas en torno a la gestión pública ambiental e intercultural y los complejos procesos de interacción entre Estado y sociedad, tanto a nivel nacional como en escenarios locales.

Los dos primeros textos de Torres y Narváez (2011) establecen y analizan un marco macro nacional y general de referencia en torno a políticas públicas, interculturalidad y derechos. Evidencian los cambios y los retos al mismo tiempo que enfrenta el Estado para dar cuenta del entorno que gobierno y de los instrumentos jurídico-institucionales y administrativos que posee para procesar las demandas de los distintos actores. Los tres textos restantes (López y los colectivos de investigadores de la Fundación Futuro Latinoameciano FFLA y Ecociencia) plantean experiencias específicas y actuales de diálogo, participa- 
ción y tratamiento de la conflictividad en torno a la gestión de territorios, áreas ecológicamente frágiles, cuencas hidrográficas y zonas sensibles atravesadas por problemas de inseguridad como lo es la frontera colombo-ecuatoriana.

Y finalmente el libro tercero Conflictos socioambientales y Estado. La búsqueda de nuevos enfoques y prácticas que es escrito por el Equipo Técnico de la Subsecretaría de Diálogo Social de la Secretaría de Pueblos, Movimientos Sociales y Participación Ciudadana (SPPC), recoge una experiencia acumulada de los últimos tres años, y que muestra los inéditos avances que en esta materia van construyendo instituciones públicas como esta secretaría de Estado, la misma que va desde la formulación de un marco conceptual y metodológico, hasta la constitución y formación de equipos técnicos a nivel de las distintas provincias y su accionar en distintos escenarios conflictivos existentes en el país. La compilación de información, la elaboración de diagnósticos, el acompañamiento y facilitación de procesos de diálogo al interior del Estado y en los territorios, con distintos actores y grupos de interés, y la construcción de acuerdos basados en el respeto, la observancia de los derechos colectivos y las demandas de las organizaciones sociales, son algunas de las premisas y referencias que han orientado y orientan la acción de la SPPC.

Como lo señala en el prólogo el primero de los tres libros, "el sentido de socializar estas reflexiones -hasta hoy muy endógenas a cada institución participante- e invitar a las organizaciones sociales urbanas y rurales del país, a la academia, a las ONG y en particular a las instituciones públicas nacionales y locales a involucrarse en estos ejercicios de reflexión, diálogo, debate, conversaciones y un mayor conocimiento de las experiencias e iniciativas en marcha en torno a los conflictos socioambientales en distintas partes del país, es contribuir a procesos más democráticos, participativos e incluyentes en el diseño, ejecución y evaluación de las políticas públicas, y en particular de aquellas relacionadas a la gestión territorial y ambiental". Con estas publicaciones la Universidad Politécnica Salesiana y Editorial Universitaria Abya-Yala en particular dan continuidad a su apoyo a la investigación y producción de trabajos en esta temática de los conflictos socioambientales producidas desde los años noventa. 


\section{Bibliografía}

Bhargava, Rajeev. Individualism in Social Science: Forms and Limits of a Methodology. Oxford: Clarendon Press, 1992.

Bobbio, Norberto. Estudios de Historia de la Filosofía. De Hobbes a Gramsci. Madrid: Editorial Debate, 1985.

Carrillo, Jenny et al. Conflictos Socioambientales y Estado. La búsqueda de nuevos enfoques y prácticas. Quito: Universidad Politécnica Salesiana, Secretaría de Pueblos, Movimientos Sociales y Participación Ciudadana y Editorial Abya-Yala, 2011.

Dahrendorf, Ralf. Las clases sociales y su conflicto en la sociedad industrial. Madrid: Ediciones Rialp, 1979.

Dahrendorf, Ralf. El conflicto social moderno: ensayo sobre la política de la libertad. Madrid: Mondibérica, 1990.

Galtung, Johan. Peace by Peaceful Means: Peace and Conflict, Development and Civilization. Oslo: International Peace Research Institute, 1996.

Galtung, Johan. "Violencia, Paz e Investigación sobre la Paz", en Galtung, Johan (editor), Sobre la Paz. Barcelona: Fontamara, 1985.

Giddens, Anthony. La Política del Cambio Climático. Madrid: Alianza Editorial, 2010.

Giddens, Anthony. La constitución de la sociedad: Bases para la teoría de la estructuración. Buenos Aires: Amarrortu, 2006.

Giddens, Anthony. La estructura de clases en las sociedades avanzadas. Madrid: Alianza, 1994.

Gleick, Peter H. Water and Conflict. Fresh water resources and international security. En International Security, Vol.18, No.1. Cambridge, MA: The MIT Press, 1993. Págs. 79-112.

Grathoff, Richard (edit.) The Theory of Social Action: The Correspondence of Alfred Schutz and Talcott Parsons. Bloomington: Indiana University Press, 1978.

Hobbes, Thomas. Leviatán o la Materia, Forma y Poder de una República, Eclesiástica y Civil. Buenos Aires: Fondo de Cultura Económica, 2005. 
Holst, Luitgard-Berenike. La propuesta kantiana de paz: un comentario sobre el debate actual en torno a la paz democrática. En Navarro, Pablo E.(comp.) La relevancia del derecho: ensayos de filosofía jurídica, moral y política. Barcelona: Editorial Gedisa, 2002. pp. 291-320.

Homer-Dixon, Thomas. On the Threshold. Environment Changes as Causes of Acute Conflict". En International Security, Vol. 16, № 2, Cambridge: MIT Press, 1991.

Homer-Dixon, Thomas. Environment, Scarcity and Violence. Princeton: Princeton University Press, 1999.

Kant, Immanuel. Sobre la paz perpetua. Madrid: Alianza, 2002.

Naff, Thomas Naff y Matson, Ruth C. Water in the Middle East: conflict or cooperation?. Boulder: Published in cooperation with the Middle East Research Institute, University of Pennsylvania, Westview Press, 1984.

Ortiz-T., Pablo. "Aproximación conceptual a los conflictos socioambientales" (CSA), en Varios, Mirar los Conflictos Socioambientales. Una relectura de conceptos, métodos y contextos. Quito: Universidad Politécnica Salesiana, Secretaría de Pueblos, Movimientos Sociales y Participación Ciudadana y Editorial Abya-Yala, 2011.

Ortiz-T., Pablo. Capitalismo extractivo, minería y derechos: ¿pueden cohabitar? en Revista Íconos No. 39. Quito: FLACSO,2011. Pp. 11-23

Ortiz-T., Pablo. Guía Metodológica para la Gestión Participativa de Conflictos Socioambientales. Quito: Forest, Trees and People Programme/FAO, Embajada Real de los Países Bajos, Comunidec, Abya-Yala, 2001.

Orellana, René y Carlos Crespo . Conflictos Ambientales. Dos Casos: Agua y Territorio. Cochabama: CERES, 1999.

PADH. Develando el desencanto. Informe sobre derechos humanos Ecuador 2010. Quito: Universidad Andina Simón Bolívar/Abya-Yala, 2011.

Parsons, Talcott. La estructura de la acción social. Madrid: Ediciones Guadarrama, 1968.

Renner, Michael. Fighting for Survival. Environmental Decline, Social Conflict and the New Age of Insecurity. New York: WW.Norton \& Co., 1996. 
Smith, Adam. Investigación sobre la naturaleza y causas de la riqueza de las naciones. Barcelona: Oikos-Tau, 1987.

Smith, Adam. Essays on Philosophical Studies. Oxford: Oxford University Press, 1980.

Terán, Juan Fernando. Desarrollo, recursos naturales y conflictos socioambientales en el capitalismo del Siglo XXI, en Varios, Mirar los Conflictos Socioambientales. Una relectura de conceptos, métodos y contextos. Quito: Universidad Politécnica Salesiana, Secretaría de Pueblos, Movimientos Sociales y Participación Ciudadana y Editorial Abya-Yala, 2011.

Torres, Víctor Hugo, María José Narváez y Victor López. Conflictos Socioambientales, Políticas Públicas y Derechos. Aproximación a un Debate. Quito: Universidad Politécnica Salesiana, Secretaría de Pueblos, Movimientos Sociales y Participación Ciudadana y Editorial Abya-Yala, 2011.

Wallensteen, Peter. Understanding Conflict Resolution. War, Peace and Global System, 2a. ed., London: Sage Publications, 2007. 